\title{
Hysteroscopic Evaluation of the Uterine Cavity in Women with First-Trimester Missed Miscarriage: Case Series
}

\author{
Altraigey $\mathrm{A}^{* 1}$, Nosseir $\mathrm{M}^{1}$, Abbas $\mathrm{A}^{2}$, Mohamed $\mathrm{M}^{3}$, Elsayed $\mathrm{M}^{3}$, Anwar $\mathrm{N}^{3}$, and Zineldin $\mathrm{Y}^{3}$ \\ ${ }^{1}$ Department of Obstetrics and Gynaecology, Benha University, Benha, Egypt \\ ${ }^{2}$ Department of Obstetrics and Gynaecology, Assiut University, Assiut, Egypt \\ ${ }^{3}$ Department of Obstetrics and Gynecology, Armed Forces Hospitals Southern Region, Khamis Mushayt, Saudi Arabia
}

*Corresponding author: Altraigey A, M.D., King Faisal Military City, base villa 9, Khamis Mushayt, 61961, Kingdom of Saudi Arabia - 43 Benha-Zagazig Street, Mansheyet Elnoor, Benha, 13511, Arab Republic of Egypt Tel: +966544854232,+201060885050,E-mail: ahmed.altraigey@yahoo.com, ahmed.abdelfattah@ fmed.bu.edu.eg

Citation: Altraigey A, Nosseir M, Abbas A, Mohamed M, Alsayed M, et al. (2017) Hysteroscopic Evaluation of the Uterine Cavity in Women with First-Trimester Missed Miscarriage: Case Series. J Gynecol Res 3(1): 105. doi: 10.15744/2454-3284.3.105

Received Date: January 17, 2017 Accepted Date: May 23, 2017 Published Date: May 24, 2017

\begin{abstract}
The aim of this study was to identify the value of hysteroscopy in detection of the relationship between missed miscarriage and intrauterine pathologies. The study was held at the Department of Obstetrics and Gynaecology, Benha University. It included 90 women with $1^{\text {st }}$ trimester missed miscarriage who were examined by diagnostic hysteroscopy immediately before dilatation and curettage (D\&C) and 6-8 weeks after D\&C (second look hysteroscopy). Incomplete uterine septum was found in $22.2 \%$ of cases; $70 \%$ of them had a previous history of missed miscarriage. Intrauterine adhesions were found in $6.7 \%$ of cases before D\&C and in $16.7 \%$ after D\&C. In cases of missed miscarriage either before or after D\&C, hysteroscopy is an easy and effective tool for diagnosis of the site of the sac and the presence of intrauterine pathology.
\end{abstract}

Keywords: Hysteroscopy; Uterine cavity; Missed miscarriage; Uterine septum; Intrauterine adhesions

\section{Introduction}

In 1878 J. Mathews Duncum introduced the term missed miscarriage. Cefalo et al. [1,2] defined missed miscarriage as the occurrence of fetal death in utero prior to 20 weeks gestation and the retention of the products for conception for a prolonged period of time.

Benign uterine pathologies either congenital (mullerian anomalies) or acquired (submucous myomas, endometrial polyps and synechia) can cause miscarriages [3]. However, the presence of uterine septum is the commonest congenital anomaly of the female genital tract, with an incidence of $2-3 \%$. [4].

In spite of the major advances in the imaging techniques, hysteroscopy is the gold standard tool for the endometrial cavity evaluation, since it allows direct endometrial visualization. Uterine abnormalities are well-identified aetiologies for miscarriage and can be detected as well as treated hysteroscopically, thus improving pregnancy outcomes [5].

Authors investigated the ability of hysteroscopy to identify the relationship between missed miscarriage and uterine pathology through hysteroscopy which was proven to be useful [6,7]. Furthermore, others studied the selective embryonic and chorionic biopsies performed by hysteroembryoscopy immediately before the curettage procedure and its specimens were more accurate $[7,8]$.

In this prospective study, we tried to identify the value of diagnostic hysteroscopy in detection of the relation of missed miscarriage and the intra-uterine pathology.

\section{Methods}

This case series was initiated after the approval of the local research ethics committee at Faculty of Medicine, Benha University and conducted over the period between January 2012 to December 2014 in the Department of Obstetrics and Gynaecology at Benha University Hospitals. 
90 cases of missed miscarriage, after being diagnosed by ultrasonographic study, were asked to participate in this study after receiving the study information sheet and signing full informed consent.

Patients with ultrasound findings of blighted ovum (anembryonic sac) were excluded from the study, as they are more likely to occur due to chromosomal abnormalities. Other exclusion criteria were excessive uterine bleeding and sonographically empty uterine cavity. The diagnosis of missed miscarriage was confirmed by histopathological study after D\&C.

All the basic demographic data of the study population as age, parity and the gestational age at diagnosis were recorded. No prostaglandin priming of the cervix was used to prevent haemorrhage, expulsion of the foetus, or leakage during procedure. All the patients received prophylactic antibiotics (1st generation cephalosporin) before the procedures.

Diagnostic hysteroscopy for each case was done twice. The first one was done immediately before D\&C under general anaesthesia and a second look hysteroscopy was repeated 6-8 weeks after D\&C (post-menstrual) as an office procedure.

All first diagnostic procedures were performed under general anaesthesia, while second look procedures were performed on outpatient basis. There were 2 surgeons, one conducted all $1^{\text {st }}$ hysteroscopy and other for all $2^{\text {nd }}$ look hysteroscopy.

Hysteroscopy was carried out in an operating theatre using a rigid microhysteroscope with continuous-flow surgical sheath with a diameter of $7 \mathrm{~mm}$ and a $3.7-\mathrm{mm}, 30^{\circ}$ telescope.

Vaginal wash with betadine solution was done. We used saline as the distension medium. To prevent excessive distension of the uterine wall, intrauterine pressure of saline solution was limited to $50 \mathrm{~mm} \mathrm{Hg}$ with an electronically controlled pump (Karl Storz, Tuttlingen, Germany). Traction on the cervix was done with a multiple-tooth tenaculum then dilatation till Hegar 7.

After passage of the hysteroscopy beyond the internal os, we waited until the uterus become distended causing the decidua parietalis to separate from the decidua capsularis.

The tubal angles, fundus, anterior, posterior and lateral walls of the uterus were inspected in order. After retracting instruments, careful standard D\&C was performed.

Second look hysteroscopy was conducted 6-8 weeks later (postmenstrual) using normal saline for uterine distension. The hysteroscopy tip was positioned in the vaginal introitus, the labia being widened by fingers. The vagina was distended with saline. The scope was used to visualize the posterior fornix and the external cervical os. When it became visible, the scope was moved forward carefully to the internal os and then the uterine cavity. At this stage it was important to reduce lateral movements to decrease patient pain and discomfort.

The primary outcome measured was the ability to diagnose the presence of any uterine pathology. Secondary outcomes were the incidence of intrauterine adhesions after D\&C and the occurrence of any complications.

The data were collected and analyzed using the Statistical Package for Social Science (SPSS Inc., Chicago, version 21). The continuous variables were presented in terms of mean and standard deviation. Dichotomous variables were presented as number and percentage.

\section{Results}

The basal demographic criteria of the study group are shown in Table 1, including age, body mass index, parity and gestational age at the time of hysteroscopy (Table 1).

\begin{tabular}{|c|c|}
\hline Variables & Mean \pm SD \\
\hline Maternal age (years) & $27.25 \pm 4.08$ \\
\hline BMI $\left(\mathrm{Kg} / \mathrm{m}^{2}\right)$ & $26.35 \pm 3.6$ \\
\hline Parity & $2.5 \pm 1.5$ \\
\hline Gestational age (weeks) & $10.85 \pm 2.4$ \\
\hline
\end{tabular}

Table 1: The demographic characteristics of the study participants

The main hysteroscopic findings are listed in (Table 2). The study included 90 women; incomplete septum was found in 20 cases $(22.2 \%)$. In 10 cases of them, the sac was found to be implanted on the septum and in one case the sac was partially implanted on the septum. Of these cases, 14 (70\%) had history of previous missed miscarriage and $2(10 \%)$ had history of preterm labour.

Intrauterine adhesions were found in 6 cases (6.6\%). Of these, $4(66.7 \%)$ had previous history of missed miscarriage (Table 2 ).

Three cases were diagnosed to have granuloma-like lesions (3.3\%). Directed biopsy from these lesions were taken and diagnosed histopathologically as chronic non-specific endometritis. Two of them had history of previous missed miscarriage and the other one had history of incomplete miscarriage. 


\begin{tabular}{|c|c|}
\hline Results & Number (\%) \\
\hline Incomplete septum & $20(22.2 \%)$ \\
\hline Intrauterine adhesions & $6(6.7 \%)$ \\
\hline Granuloma-like lesion & $3(3.3 \%)$ \\
\hline Scar pregnancy & $1(1.1 \%)$ \\
\hline Failed assessment & $2(2.2 \%)$ \\
\hline Normal cavity & $58(64.4 \%)$ \\
\hline
\end{tabular}

Table 2: The results of hysteroscopic evaluation of the uterine cavity before dilatation and curettage

In one case, the sac was found to be implanted on a scar of previous caesarean section. In two cases hysteroscopic interpretation was difficult due to the presence of black altered blood in the uterine cavity, but 6-8 weeks after D\&C second look hysteroscopy showed absolutely normal uterine cavities.

Second look hysteroscopy after 6-8 weeks from D\&C revealed the presence of intrauterine adhesions in 15 cases (16.7\%). Of these cases, 3 cases were diagnosed to have mild adhesions before D\&C. according to the European Society of Hysteroscopy (ESH) Classification, [9] the intrauterine adhesions were of mild type in 12 cases and of moderate type in 3 cases. In the last 3 cases, D\&C was repeated due to the presence of retained products of conception.

There were no intraoperative or postoperative complications among the entire operative or the $2^{\text {nd }}$ look procedures. All the patients had smooth recovery and postoperative period.

\section{Discussion}

In our study, 22.2\% of missed miscarriage cases had an incomplete septum. Among them, history of previous missed miscarriage in 14 cases (70\%) and preterm labour in 2 cases (10\%) [6]. It is concluded that the presence of incomplete uterine septum is a major contributing factor in missed miscarriage aetiology as they had 14 out of 60 cases of missed miscarriage with incomplete septum (23.3\%) and 10 of them have had a missed miscarriage in the past $(71.4 \%)$.

It is well known that any uterine abnormalities either congenital or acquired may play a role in the aetiology of spontaneous, recurrent or missed miscarriages. The patho-physiological bases by which these abnormalities cause pregnancy loss are not completely understood. One suggestion was the poor blood supply to the septum which may lead to poor implantation dynamics $[10,11]$. Moreover, foetal loss may be due to the septal space-occupying effect and impaired placentation can cause foetal growth restriction [12].

Also, missed miscarriage may play aetiology of intra-uterine pathology either by its pathogenesis (necrosis and inflammation) or by the complications after curettage [13].

Buttram, 1983 [14] found that poor vascularisation of the septum was implicated as the reason for defective implantation. Also, [15] Corson, 1992 found that pregnancy losses with septate uteri classically occur between 8-16 weeks. He concluded that poor implantation dynamics because of reduced blood supply to the septum generally has been accepted as causal but without actual verification.

This agrees with [16] who used ultrasound to locate the implantation site in 12 cases of pregnancies in septate uteri. They found that all pregnancies that aborted showed septal implantation and in all pregnancies that did not abort, implantation occurred in the lateral uterine wall. Also, [17] suggested that poor vascularisation of the septum is even poorer in cases of partial septum and therefore, a lower chance to reach term exists in such gestations.

Intrauterine synechiae is a possible complication of D\&C and its incidence has been reported to range from 15\% to 40\% [18,19]. The frequency of intrauterine adhesions may vary according to the curettage technique, preoperative and postoperative quality of care, gestational age, patient's constitutional characteristics and clinical complications following miscarriage (particularly infections) [20].

Lancent and Kessle [21] reported that approximately two thirds of patients suffering from intrauterine adhesions had a previous miscarriage ( $60 \%$ for spontaneous and $17.8 \%$ for induced miscarriages). Although [20] found the prevalence of $37.6 \%$ of the women subjected to curettage following missed miscarriage had intrauterine adhesions, they concluded that there is no firm evidence to justify carrying out routine diagnostic hysteroscopy following miscarriage evacuation. The prevalence of intrauterine adhesions was also lower in some previous studies: 25.0\% [22] and 30.2\% [23].

In this study, intrauterine adhesions do not seem to occur as frequent as was reported after missed miscarriage. Our results showed that intrauterine adhesions were found in $6.7 \%$ of patients before $\mathrm{D} \& \mathrm{C}$, which may predispose to defective implantation of the sac. Also the intrauterine adhesions were detected in $16.7 \%$ of cases with second look hysteroscopy 6-8 weeks after D\&C 
Methodological techniques differences may account for these discrepancies especially the diagnostic tools and classifications used for describing the intrauterine adhesions. Moreover, the mean time elapsed between curettage and hysteroscopy in the present study was only $6.2 \pm 1.2$ weeks, whereas it is expected that some adhesions may need longer periods of time for the adhesion formation to be established, especially thick fibrous adhesions.

Adoni et al. [18] in their prospective study on 120 patients performed hysterosalpingography 6 to 8 weeks after miscarriage; they found intrauterine adhesions in $13 \%$ of the patients. [6] demonstrated intrauterine adhesions in only $16.7 \%$ of the patients on performing diagnostic hysteroscopy 8 to 12 weeks after D \& C.

Researchers tested the role of hysteroscopy in improving the karyotyping for early missed miscarriages [8] concluded that direct hysteroembryoscopic biopsies were more accurate than the direct curettage material. In contrast, [24] found that direct hysteroscopic sampling did not improve the sensitivity of cytogenetics for aneuploidy detection when compared with conventional suction D\&C.

So, hysteroscopy has good clinical and functional results in dealing with a wide scope of pregnancy related complications such as pregnancy on top of intrauterine device, retained trophoblastic tissue, persistent molar tissue, scar pregnancy, embryoscopy and osseous metaplasia [25].

In conclusion, diagnostic hysteroscopy in cases of missed miscarriages before $\mathrm{D} \& \mathrm{C}$ is an easy, safe and effective tool for diagnosis of the site of implantation of the sac and the presence of intrauterine pathology either congenital or acquired. In the meanwhile, second look hysteroscopy after D\&C also reduces the idea that intrauterine adhesions are a common complication after curettage.

Based on the results of this, further larger comparative trials with adequate sample size and randomization are needed to prove our results.

\section{Author Contributions}

All the authors contributed in study design, data collection, analysis, interpretation and statistical analysis. They were all responsible about the procedures done after patient recruitment. Finally, the authors shared in manuscript preparation.

\section{Declaration of interest}

The authors report no declarations of interest.

\section{Impact statement}

It is well-known that the hysteroscopy is the gold standard tool for exploring the uterine cavity for any pathology. We put a hypothesis that presence of intrauterine pathology can lead to occurrence of missed miscarriage through disturbing the normal vascularity and development of the growing gestational sac. So, we conducted this case series which showed that the presence of intrauterine pathology like septum or adhesions may lead to missed miscarriage and sometimes to recurrence. There are supporting articles in the literature with the same findings. As our case series is pilot and of small sample size. If the same findings were supported by larger powered and randomized trials, this can help in producing novel protocol for management of missed miscarriage especially the recurrent type.

\section{Reference}

1. Pridjian G, Mownad AH (1989) Missed abortion: Still appropriate terminology? Am J Obstet Gynecol 161: 261-2.

2. Cefalo RC (1983) Missed abortion and antepartum foetal death. In: Wiley Blackwell, Protocols for high-risk pregnancies: 2nd Edition, United States of America.

3. Propst AM, Hill JA (2000) Anatomic factors associated with recurrent pregnancy loss. Semin Reprod Med 18: 341-50.

4. Cogendez E, Dolgun ZN, Sanverdi I, Turgut A, Eren S (2011) Post-abortion hysteroscopy: a method for early diagnosis of congenital and acquired intrauterine causes of abortions. Eur J Obstet Gynecol Reprod Biol 156: 101-4.

5. Demirol A, Gurgan T (2004) Effect of treatment of intrauterine pathologies with office hysteroscopy in patients with recurrent IVF failure. Reprod Biomed Online 8: 590-4.

6. Golan A, Schneider D, Avrech O, Raziel A, Bukovsky I, et al. (1992) Hysteroscopic findings after missed abortion. Fertil Steril 58: 508-10.

7. Philipp T, Kalousek DK (2002) Generalized abnormal embryonic development in missed abortion: embryoscopic and cytogenetic findings. Am J Med Genet 111: 43-7.

8. Ferro J, Martínez MC, Lara C, Pellicer A, Remohí J, et al. (2003) Improved accuracy of hysteroembryoscopic biopsies for karyotyping early missed abortions. Fertil Steril 80: 1260-4.

9. Wamsteker K (1990-1991) ESH Classification of intrauterine adhesions (IUA) - grade extent of intrauterine adhesions. European Society of Hysteroscopy Membership Directory: 60.

10. Homer HA, Li TC, Cooke ID (2000) The septate uterus: A review of management and reproductive outcome. Fertil Steril 73: 1-14.

11. Candiani GB, Fedele L, Zamberletti D, De Virgililis D, Carinelli S (1983) Endometrial patterns in malformed uteri. Acta Eur Fertil 14: 311-8.

12. Valli E, Vaquero E, Lazzarin N, Caserta D, Marconi D, et al. (2004) Hysteroscopic metroplasty improves gestational outcome in women with recurrent spontaneous abortion. J Am Assoc Gynecol Laparosc 11: 240-4. 
13. Kuzel D, Horak P, Hrazdirova L, Kubinova K, Sosna O, et al. (2011) "See and treat" hysteroscopy after missed abortion. Minim Invasive Ther Allied Technol 20: $14-7$.

14. Buttram VC (1983) Mullerian anomalies and their management. Fertil Steril 40: 159-63.

15. Corson SL (1992) Operative hysteroscopy for infertility. Clin Obstet Gynecol 35: 229-41.

16. Fedele L, Dorta M, Brioschi D, Giudici MN, Candiani GB (1989) Pregnancies in septate uteri: Outcome in relation to site of uterine implantation as determined by sonography. AJR Am J Roentgenol 152: 781-4.

17. Heinomen PK, Saarikoski S, Pystynen P (1982) Reproductive performance of women with uterine anomalies. Acta Obstet Gynecol Scand 61: 157-62.

18. Adoni A, Palti Z, Milwidsky A, Dolberg M (1982) The incidence of intrauterine adhesions following spontaneous abortion. Int J Fertil 27: 117-8.

19. Al-Inany H (2001) Intrauterine adhesions. An update. Acta Obstet Gynecol Scand 80: 986-93.

20. Salzani A, Yela DA, Gabiatti JR, Bedone AJ, Monteiro IM (2007) Prevalence of uterine synechia after abortion evacuation curettage. Sao Paulo Med J 125: 261-4.

21. Lancet M, Kessler I (1988) A review of Asherman's Syndrome and results of modern treatment. Int J Fertil 33: 14-24.

22. Malik E, Berg C, Sterzik K, Stoz F, Rossmanith WG (2000) Reproductive outcome of 32 patients with primary or secondary infertility and uterine pathology. Arch Gynecol Obstet 264: 24-6.

23. Römer T (1994) Post-abortion hysteroscopy--a method for early diagnosis of congenital and acquired intrauterine causes of abortions. Eur J Obstet Gynecol Reprod Biol 57: 171-3.

24. Awonuga AO, Jelsema J, Abdallah ME, Berman J, Diamond MP, et al. (2010) The role of hysteroscopic biopsy in obtaining specimens for cytogenetic evaluation in missed abortion prior to suction dilatation and curettage. Gynecol Obstet Invest 70: 149-53.

25. Pérez-Medina T, Sancho-Saúco J, Ríos M, Pereira A, Argila N, et al. (2014) Hysteroscopy in pregnancy-related conditions: descriptive analysis in 273 patients. Minim Invasive Gynecol 21: 417-25.

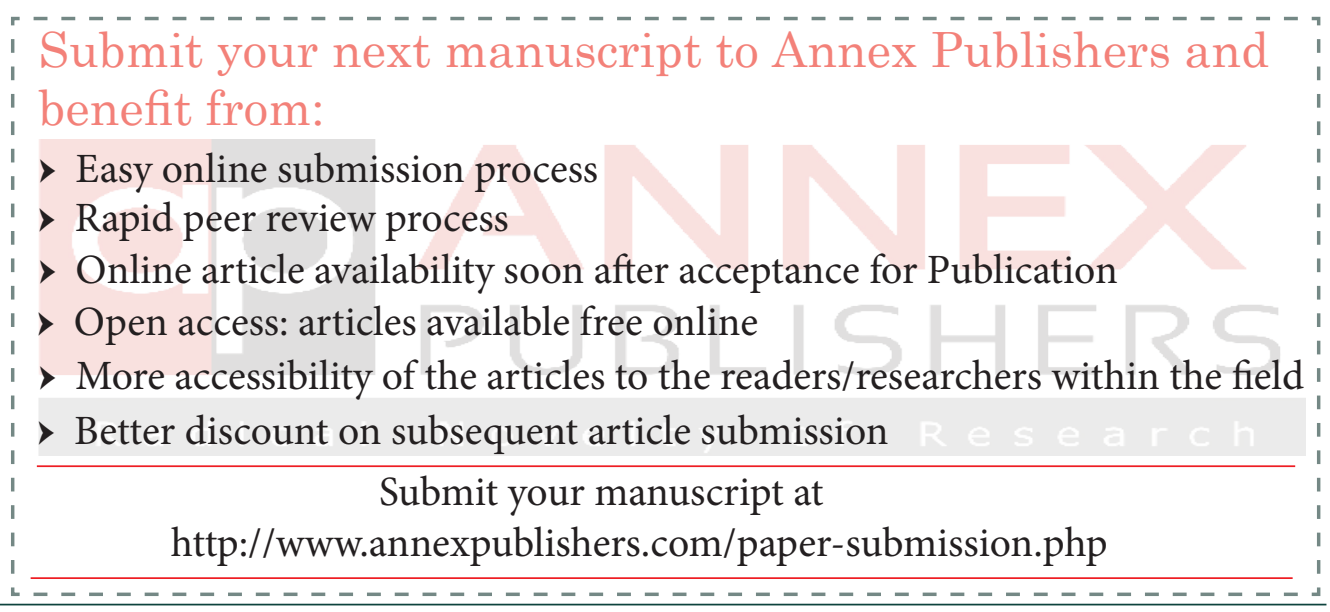

